\title{
FPGA BASED CLOCK FREQUENCY SENSOR AND RANGE ESTIMATING SYSTEM FOR TESTING MEMORIES AND PROCESSORS
}

\author{
C. Manjula \\ Oxford College of Engineering, Bengaluru, \\ Affiliated to Visvesvaraya Technological University, Belgaum, Karnataka, India

\section{Jayadevappa} \\ JSS Academy of Technical Education, Bengaluru, \\ Affiliated to Visvesvaraya Technological University, Belgaum, Karnataka, India
}

\begin{abstract}
Research on development of FPGA based Universal Digital ATE there is a strong need to sense and estimate the clock frequency \& speed of processors or memory or any digital logic system or IP core etc. Till date no such frequency sensor and estimator product is available nor is such a research work reported or published. Hence this research work is very unique with novel design and innovative approach using FPGA, providing a major breakthrough in frequency ranging and estimation domain.
\end{abstract}

Key words: Digital Pattern Generator, Clock Edge detector, Digital Logic Analyser, Frequency Measurement, ATE, FPGA.

Cite this Article: C. Manjula and D. Jayadevappa, FPGA Based Clock Frequency Sensor and Range Estimating System for Testing Memories and Processors. International Journal of Electrical Engineering and Technology, 11(5), 2020, pp. 11-21.

http://iaeme.com/Home/issue/IJEET?Volume $=11 \&$ Issue $=5$

\section{INTRODUCTION}

The following figure 1 shows the design of proposed Digital Test Pattern Generator (DTPG) implemented on Xilinx Spartan FPGA. The design and simulation results of all individual sub modules- Digital Frequency Synthesizer, Pattern Generator, Switch Matrix and DUT of the FPGA based DTPG was published in IEEE Digital Library journal [1], [2]. 
FPGA Based Clock Frequency Sensor and Range Estimating System for Testing Memories and Processors

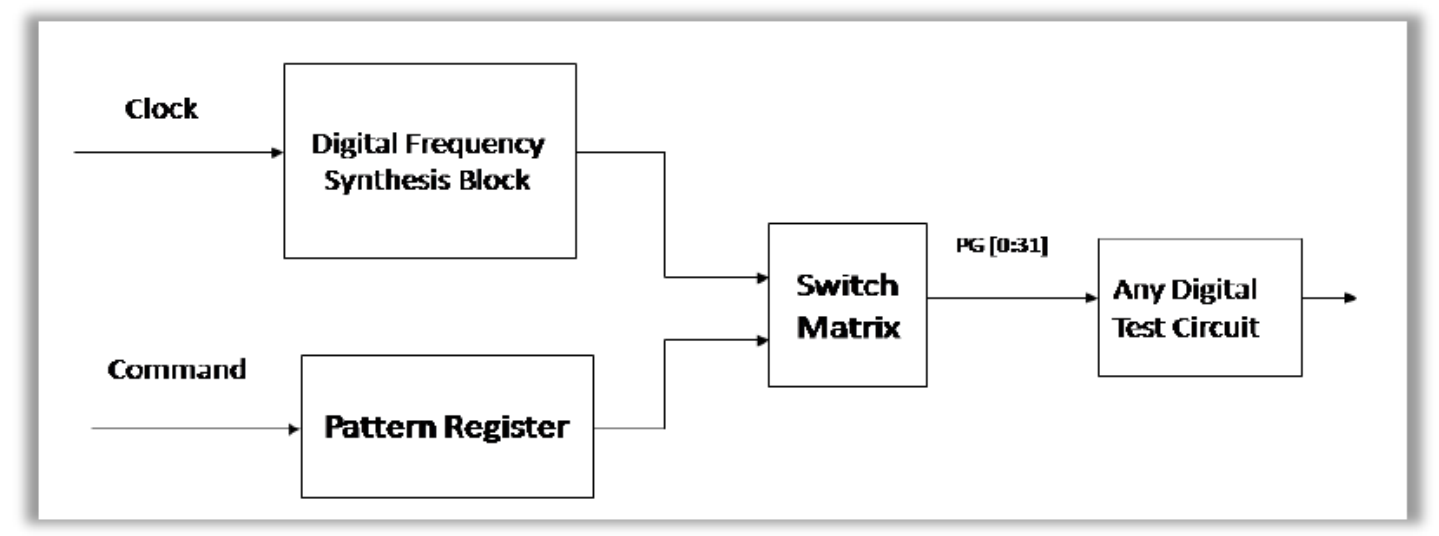

Figure 1 Digital Pattern Generator

Research Experimental set up for FPGA based ATE to test presence of processor clock and to measure or Auto Ranging of incoming clock frequency signal is described in the following section.

\subsection{Design 1: Processor Clock Signal Presence Detector using Edge Detector}

The block diagram shown in figure 2 represents the edge detector circuit to detect the presence of Processor clock. The Edge detector is designed with D flip-flop with an Inverter gate and AND gate at the output [3]. The output of the D flip-flop will be delayed and is applied to the AND GATE with the Inverted D flip-flop output Q.

Short Pulses are obtained at every edge of the input signal to the flip [flop, indicating the presence of the rising edge or a clock signal. The digital above circuit with a minor modification can be made to detect falling edge also.

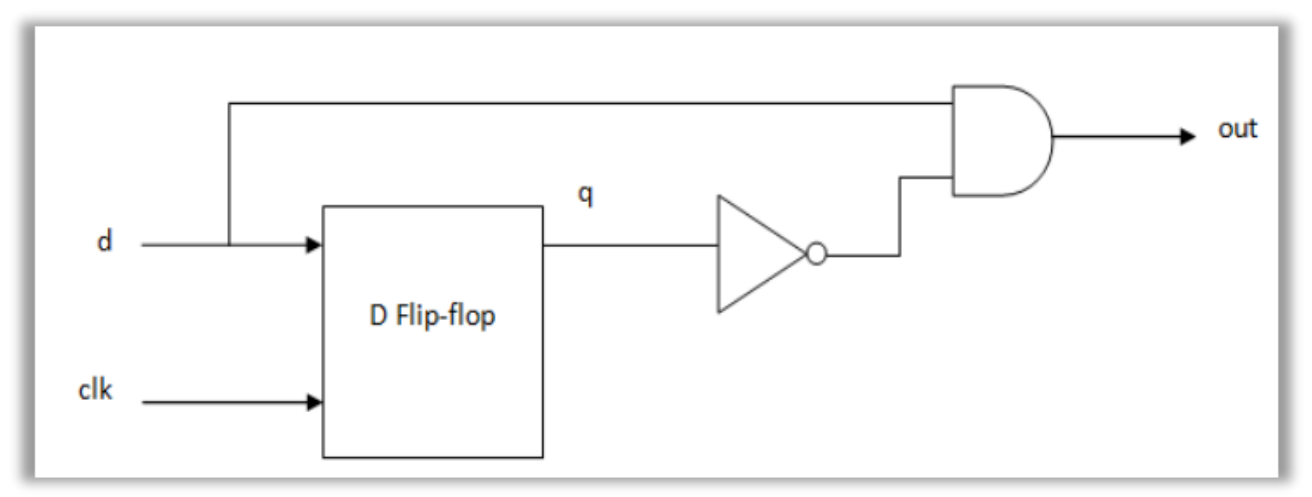

Figure 2 Edge detector circuit to detect the presence of Processor clock.

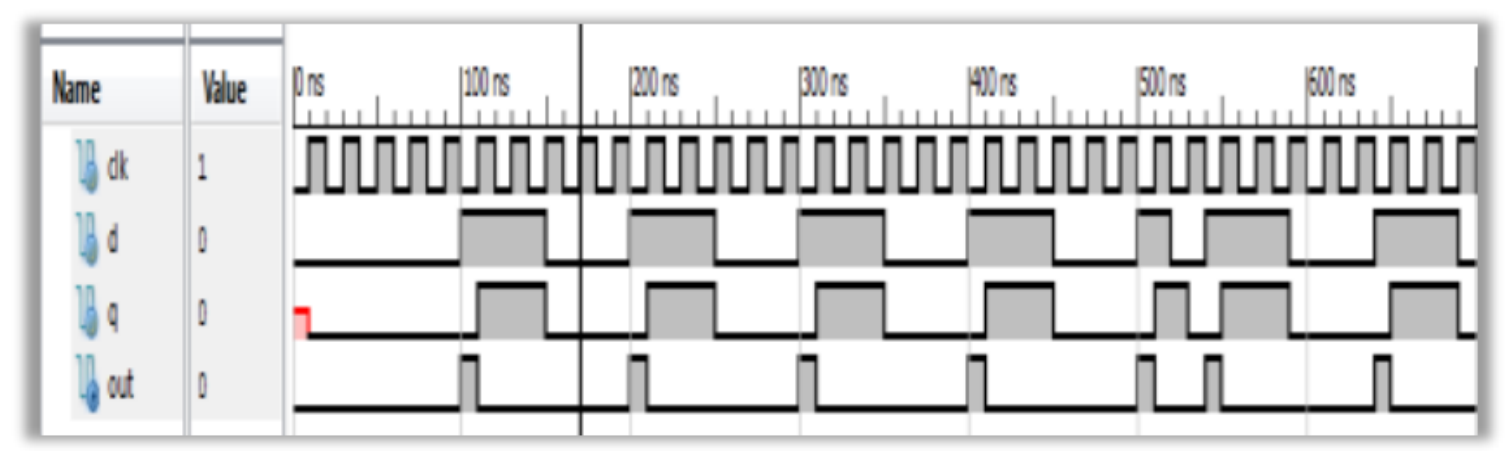

Figure 3 Simulation waveform of Edge detector circuit to detect the presence of Processor clock. 
Simulation waveform descriptions are as follows.

- Incoming Clock signal can have any suitable frequency, depending on the FPGA chip used (Set up time dependent)

- Waveforms d\& q represents the input \& output of d flip-flop.

- Out waveform above represents the output of the Edge detector, which becomes high for a small duration, depending on propagation delay of the FF \& gates, at all the positive edges of the Signal applied to Edge detector Flip flop.

- These short pulses are periodic \& hence they indicate the presence of a CLOCK signal.

- This circuit can be used to detect presence of any clock signal in Processors or Memory or any Logic Circuits.

\subsection{Design 2: FPGA based Clock Frequency Sensor and Range Estimating System}

The FPGA based Processor or Memory Clock Frequency Sensor and Range Estimating System specialty is that, this can indicate or measure any frequency range from $1 \mathrm{~Hz}$ to $1 \mathrm{GHz}$ or above. To prove the above functionality, we make use of the experimental set up depicted in figure 4, wherein variable frequencies generated at the output of DPG $-1 \mathrm{~Hz}, 10 \mathrm{~Hz}, 100 \mathrm{~Hz}$, $1 \mathrm{KHz}, 10 \mathrm{KHz}, 100 \mathrm{KHz}, 1 \mathrm{MHz}$ are applied to CRIMS for frequency measurement or range estimations using LED array strip on the FPGA demo board. The FPGA board has a crystal clock frequency of $10 \mathrm{MHz} \&$ the other lower frequencies like $1 \mathrm{MHz}, 100 \mathrm{KHz}, 10 \mathrm{KHz}, 1$ $\mathrm{KHz}, 100 \mathrm{~Hz}, 10 \mathrm{~Hz}, 1 \mathrm{~Hz}$ are derived from this Master clock, by successive clock division. The frequencies freq $1 \mathrm{MHz}$, freq $100 \mathrm{KHz}$, freq $10 \mathrm{Khz}$, freq $1 \mathrm{KHz}$, freq $100 \mathrm{~Hz}$, freq $10 \mathrm{~Hz}$, freq $1 \mathrm{~Hz}$ are the output signals that were already generated by the DPG. This can be used to emulate Processor or Memory or any Chip under test or DUT clock signal. This experimental system can detect presence of clock signal applied to any Processor or Memory or DUT \& measure the frequency or estimate the Range [4].

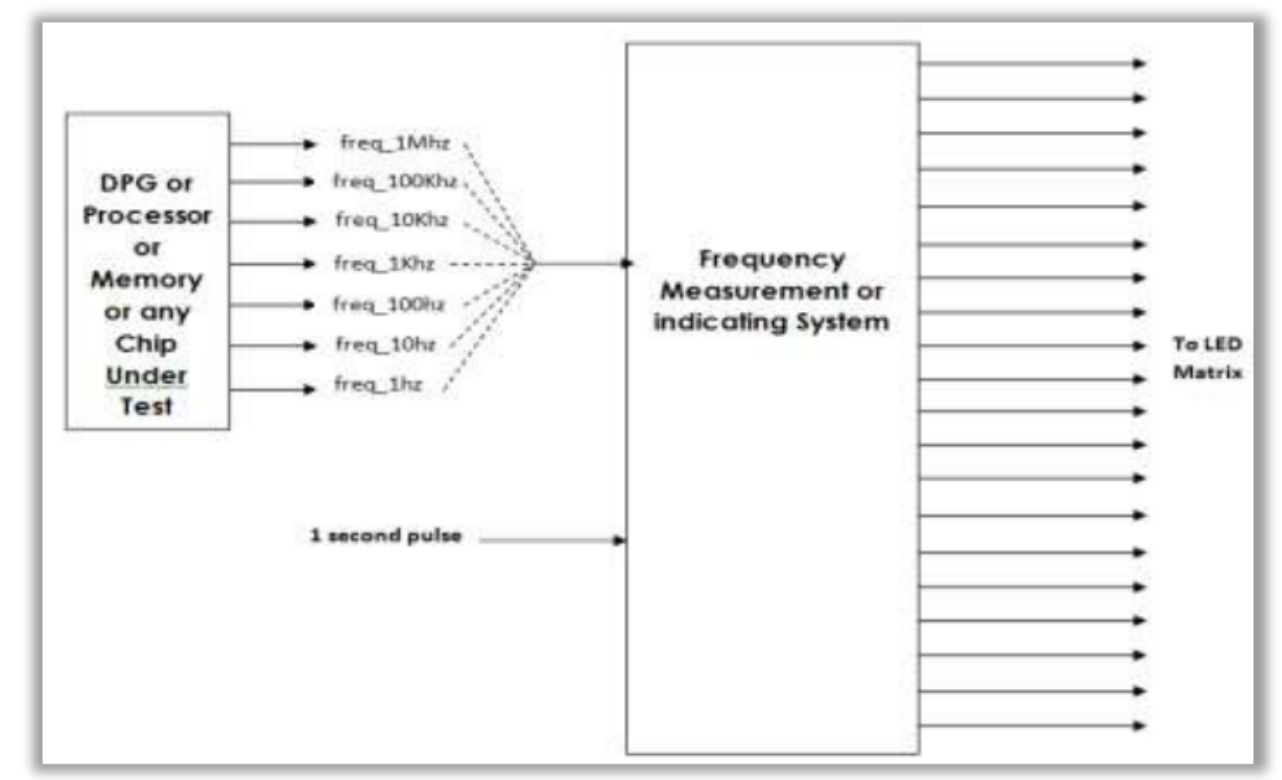

Figure 4 Clock Frequency Sensor \& Range Estimating System (FCSRE).

The number of LEDs glowing ON or OFF matrix is an indication of the frequency range of the incoming clock signal to the FCRES. If the input frequency from DPG or DUT to 
FPGA Based Clock Frequency Sensor and Range Estimating System for Testing Memories and Processors

CRIMS is $1 \mathrm{~Hz}$, then only 2 of the 20 LEDs in the LED array will toggle indicating 1 clock cycle consisting of 2 pulses \& the frequency counter counts from 00 at reset to $01 \& 10$ for the two pulses in $1 \mathrm{hz}$ incoming clock signal. Likewise, if the input frequency is $10 \mathrm{hz}$, then 4 of the 20 LEDs will toggle \& if input frequency of $100 \mathrm{hz}$, then 7 of the 20 LEDs will toggle.

For other higher frequencies, the number of LEDs out of 20 LED array, will toggle is as indicated in the table 1 . When respective clock frequency is applied, corresponding or appropriate number of LEDs must glow as per the above table, to Auto Range the incoming clock frequency signal [5], [6].

Table 1 Clock frequency, Binary count and glowing LEDs of FCSRE

\begin{tabular}{|l|l|l|l|}
\hline \multicolumn{1}{|c|}{ Incoming Clock } & \multicolumn{1}{c|}{ No. of Clock } & No. of & Maximum Binary Count \\
\hline Data_1Mhz & 1000000 & 20 & 11110100001001000000 \\
\hline Data_100Khz & 100000 & 17 & 11000011010100000 \\
\hline Data_10Khz & 10000 & 14 & 10011100010000 \\
\hline Data_1Khz & 1000 & 10 & 1111101000 \\
\hline Data_100hz & 100 & 7 & 1100100 \\
\hline Data_10hz & 10 & 4 & 1010 \\
\hline Data_1hz & 1 & 2 & 01 \\
\hline
\end{tabular}

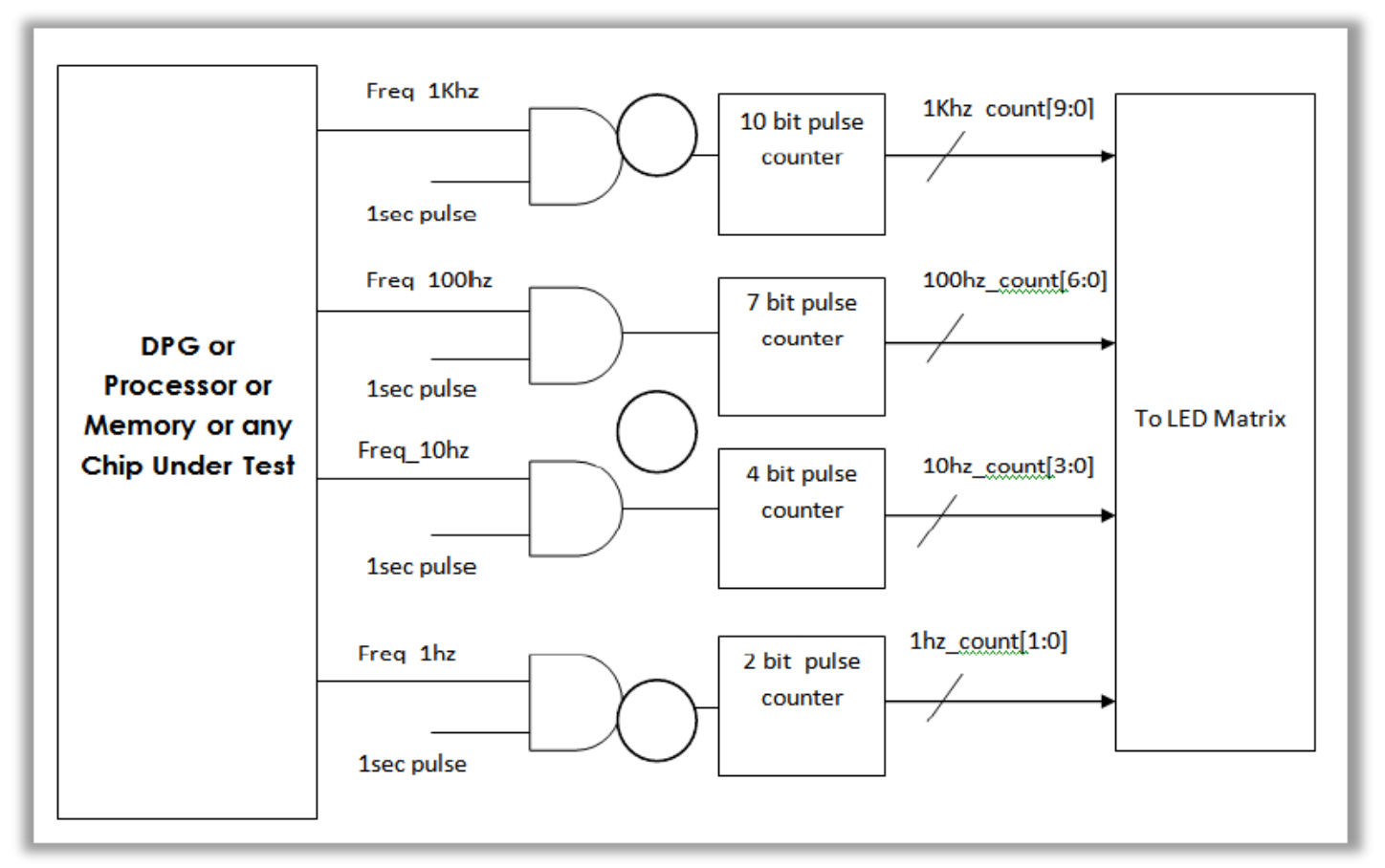

Figure 5 Clock Frequency Sensor and Range Estimating System (FCSRE).

\section{Processor with clock frequency of $1 \mathrm{~Hz}$}

- Clock is $10 \mathrm{MHz}$.

- CRIMS is applied with a single frequency at a time for auto ranging and frequency estimation.

- Freq $1 \mathrm{~Hz}$ is a $1 \mathrm{~Hz}$ signal that is generated from $10 \mathrm{MHz}$ clock by 6 times successive division by 10 .

- To estimate or measure frequency of any incoming signal is shown in the figure 5 . 
- An AND gate whose output $\mathrm{x}$ is applied with this frequency $1 \mathrm{~Hz}$ and 1 second pulse (reference time base).

- AND gate output x goes high, only when both its input becomes 1 .

- The frequency measurement strategy is to count the number of pulses in 1 second reference time.

- At the commencement of 1 second pulse the 2 bit pulse counter output $1 \mathrm{~Hz}$ counts (1:0) shows an initial count of 00 , since no pulses are counted.

- During the rising edge of the clock pulse (freq $1 \mathrm{~Hz}$ ), 2 bit pulse counter output $1 \mathrm{~Hz}$ _counts (1:0) changes from 00 to 01 .

- 01 in binary indicates a decimal value of 1 , which is equal to a number of a pulses counted by the pulse counter in 1 second time.

- Hence the incoming frequency value is estimated at $1 \mathrm{~Hz}$.

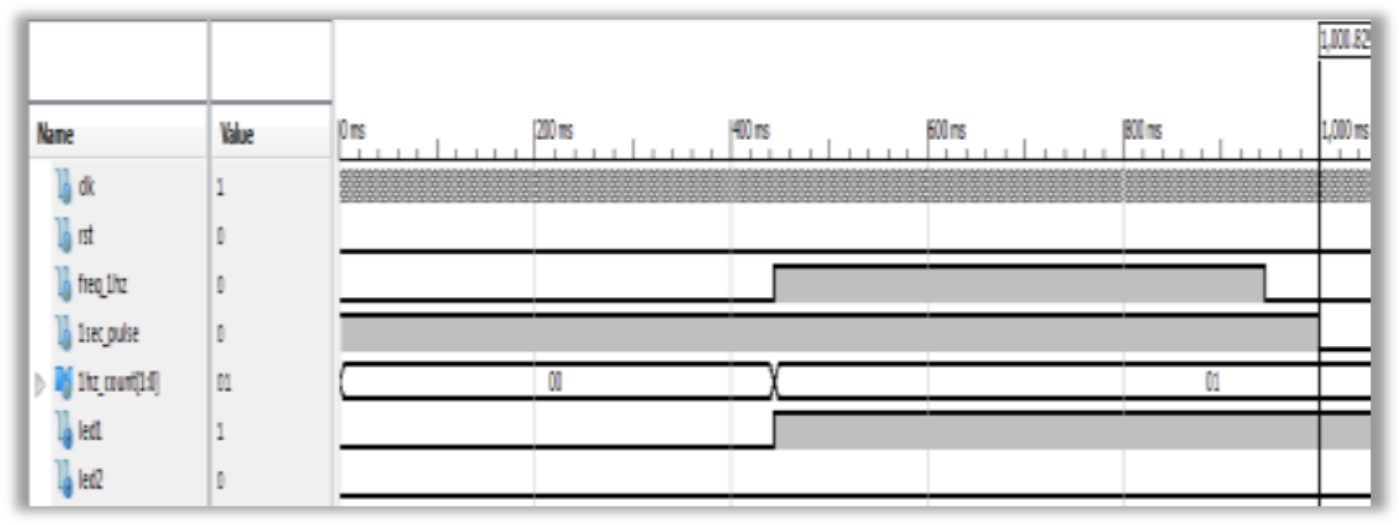

Figure 6 Simulation waveforms for the frequency of $1 \mathrm{~Hz}$.

\section{Processor with clock frequency of $100 \mathrm{~Hz}$}

- Freq $10 \mathrm{hz}$ is $100 \mathrm{~Hz}$ clock signal generated from $10 \mathrm{MHz}$ clock by 4 times successive division by 10 .

- Input signal Freq $100 \mathrm{hz}$ represents the frequency of the Processor clock.

- $100 \mathrm{~Hz}$ _count $(6: 0)$ is the output signal which indicates the no of clock cycles obtained by the clock frequency of $100 \mathrm{~Hz}$.

- Frequency of $100 \mathrm{~Hz}$ generates 100 clock cycles (1100100) in $1000 \mathrm{Ms}$ represented by signal 1Khz_count $(9: 0)$.

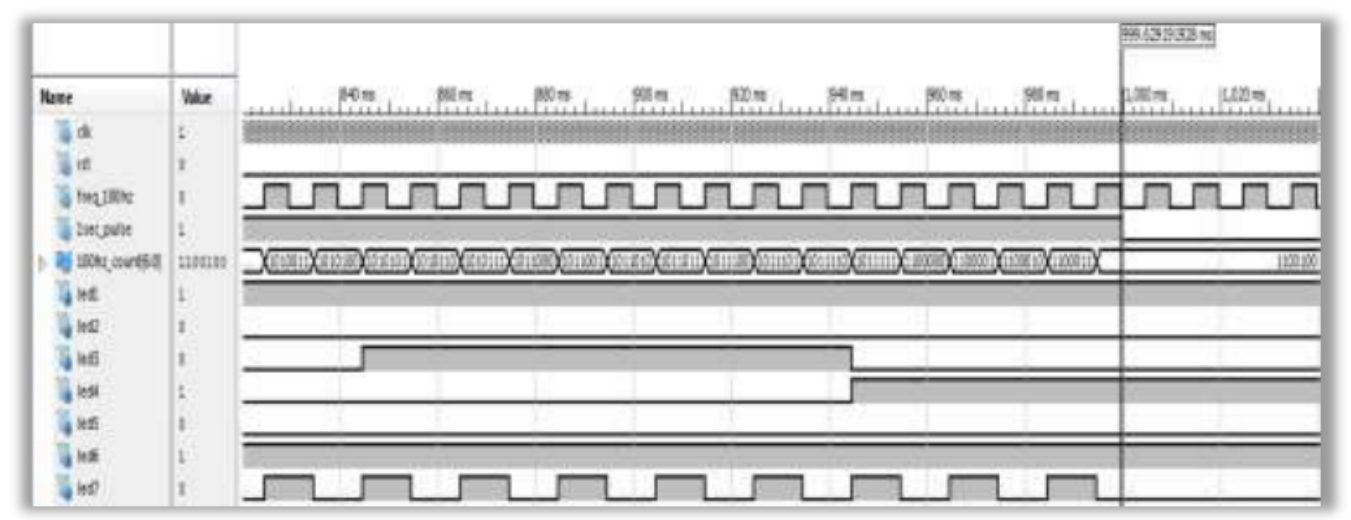

Figure 7 Simulation waveforms for the frequency of $100 \mathrm{~Hz}$. 
FPGA Based Clock Frequency Sensor and Range Estimating System for Testing Memories and Processors

\section{Processor with clock frequency of $1 \mathrm{KHz}$ clock}

- Freq $10 \mathrm{hz}$ is $1 \mathrm{KHz}$ clock signal generated from $10 \mathrm{MHz}$ clock by 3 times successive division by 10 .

- Input signal Freq_1Khz represents the frequency of the Processor clock [7].

- 100hz_count (9:0) is the output signal which indicates the no of clock cycles obtained by the clock frequency of $1 \mathrm{KHz}$.

- Frequency of $1 \mathrm{KHz}$ generates 1000 clock cycles (1111101000) in 1000Ms represented by signal 1Khz_count (9:0).

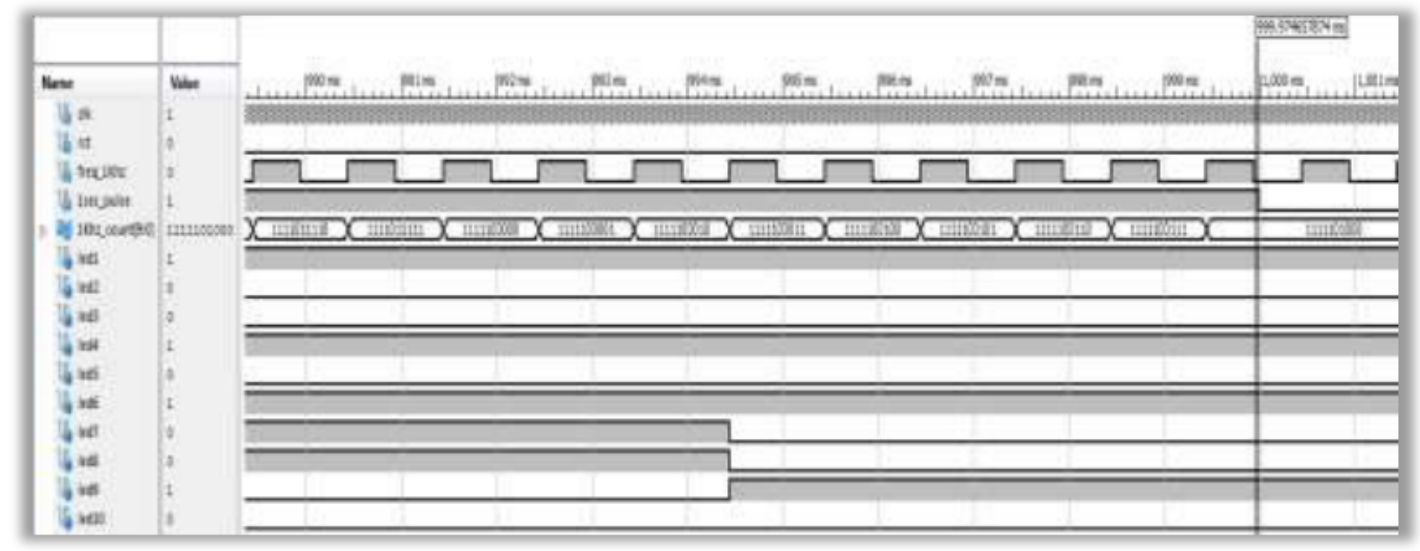

Figure 8 Simulation waveforms for the frequency of $1 \mathrm{KHz}$.

The following diagrams illustrates the FPGA [8], [9] demonstration of Clock Detector and Frequency Sensor and Range Estimator.

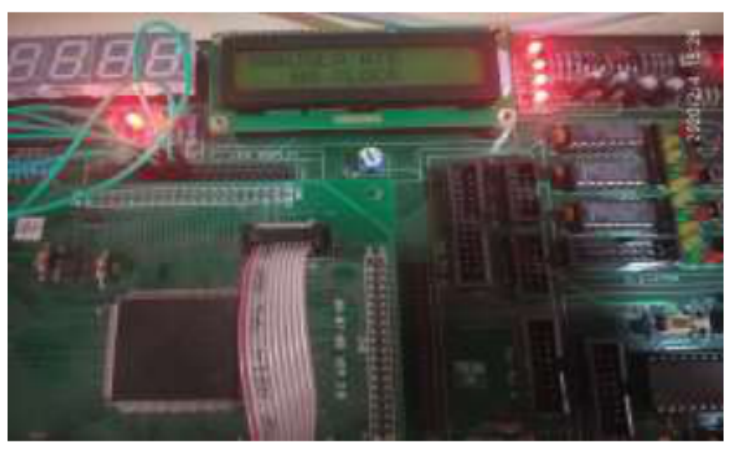

(a)

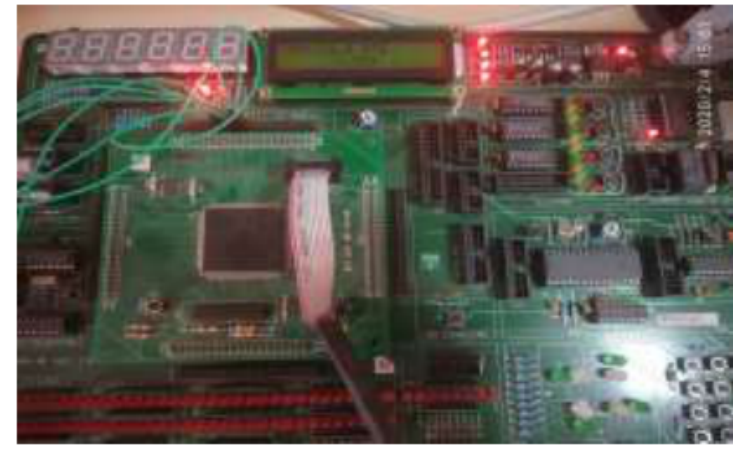

(b)

Figure 9 (a) No Clock detected by the Clock detector and (b) The Clock detected by the Clock detector.

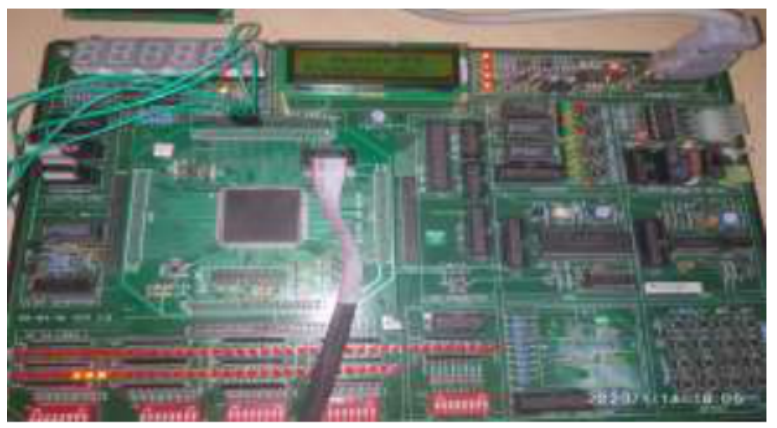

(a)

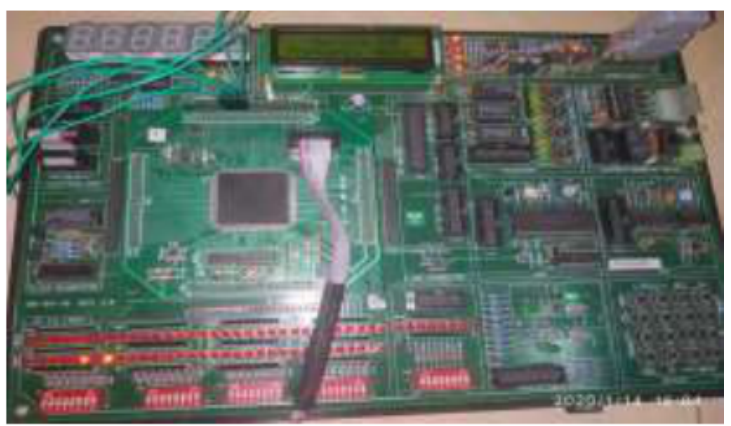

(b)

Figure 10 (a) The incoming clock frequency is in the range of $1 \mathrm{~Hz}$ and (b) The incoming clock frequency is in the range of $100 \mathrm{~Hz}$. 


\section{DLA TEST METHODOLOGY}

To verify DLA functionality, already verified DPG programmable outputs is directly interfaced to DLA wherein both asynchronous and synchronous data from DPG can be loaded to DLA. The DLA output will be in terms of asynchronous values and synchronous waveforms. The DPG programmable outputs can be changed using command and corresponding changes can be observed in the DLA output [10], [11] \& [14 - 20].

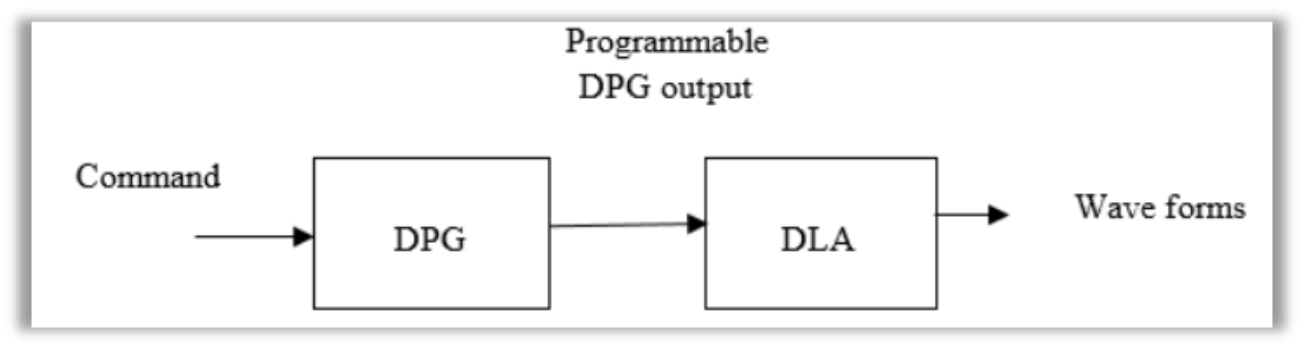

Figure 11 The experimental and test set up to verify DLA functionality.

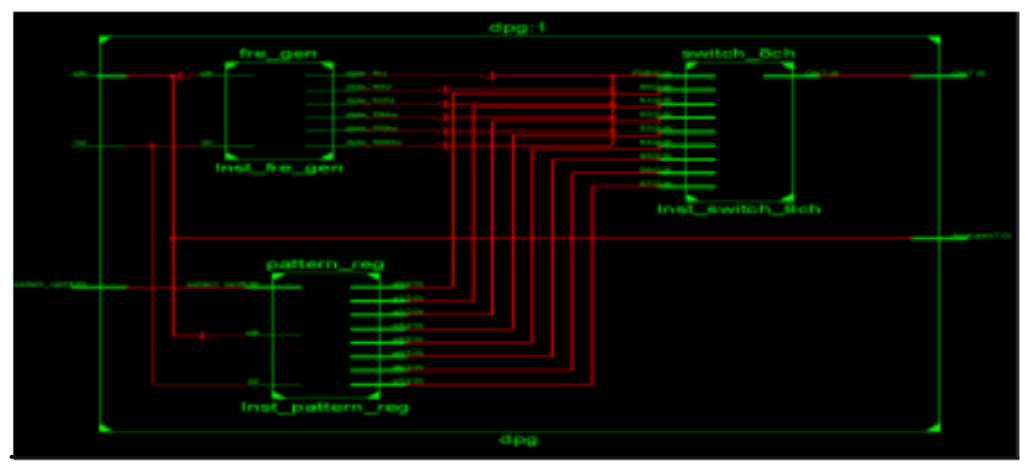

Figure 12 RTL Schematic of Digital Pattern Generator.

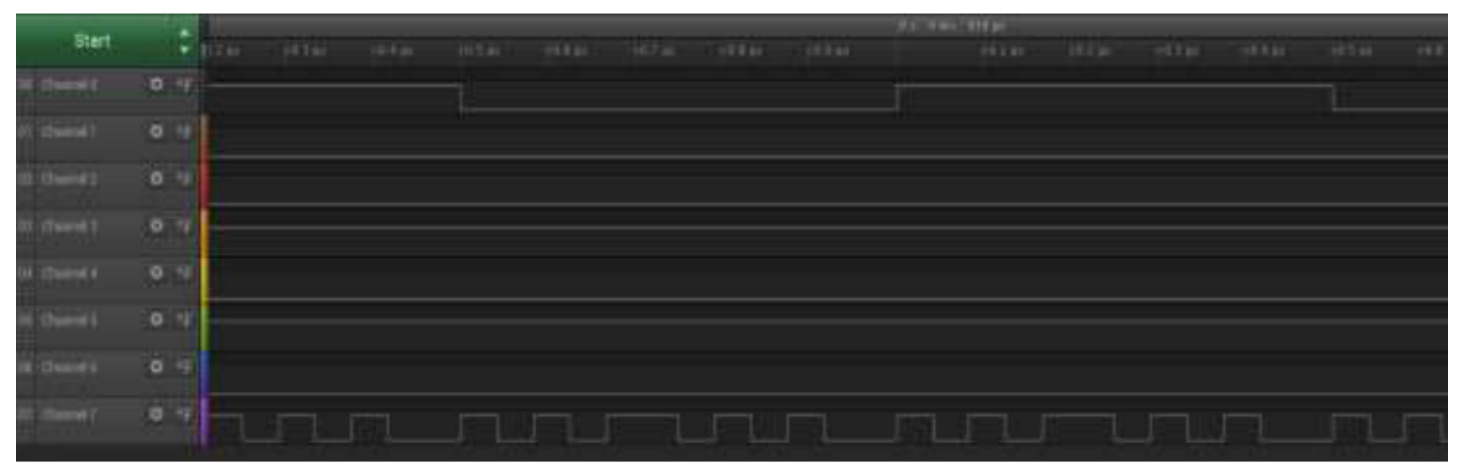

Figure $131 \mathrm{MHz}$ frequency on Channel 0.

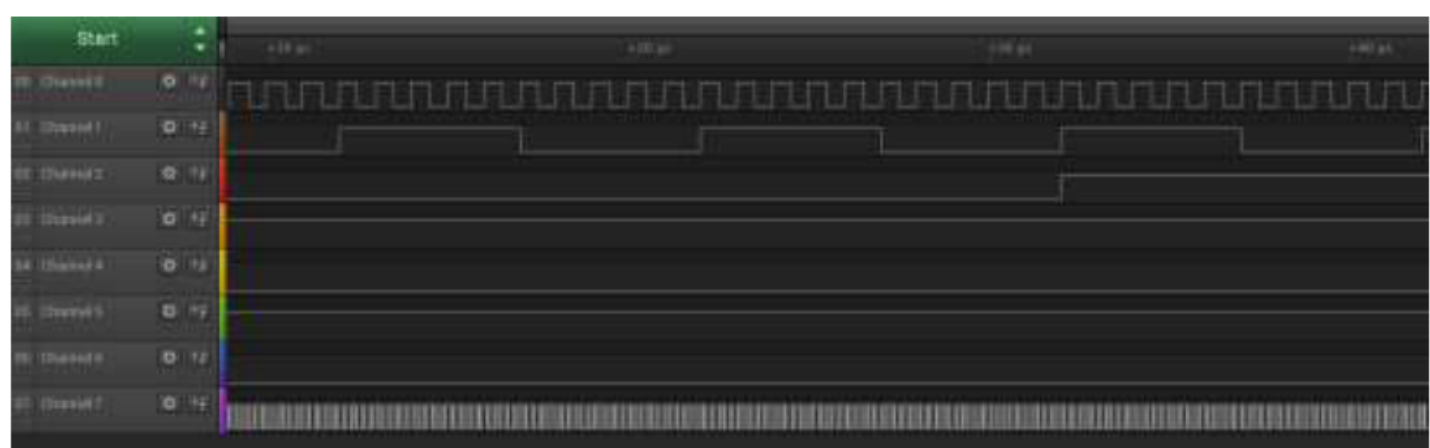

Figure $1410 \mathrm{KHz}$ frequency on Channel 1. 
FPGA Based Clock Frequency Sensor and Range Estimating System for Testing Memories and Processors

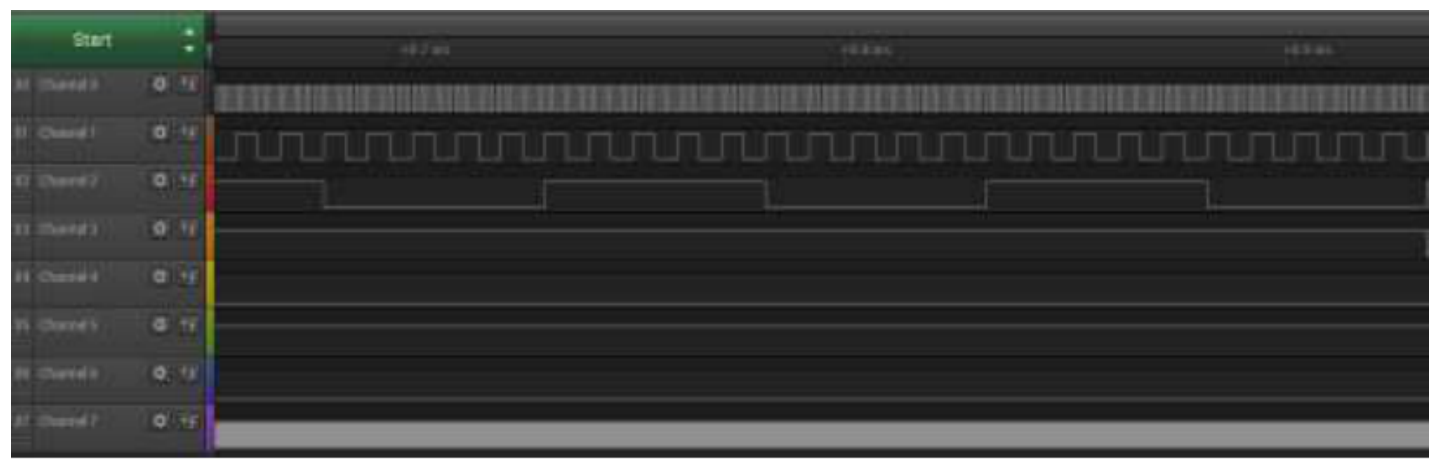

Figure $151 \mathrm{~K} \mathrm{~Hz}$ frequency on Channel 2.

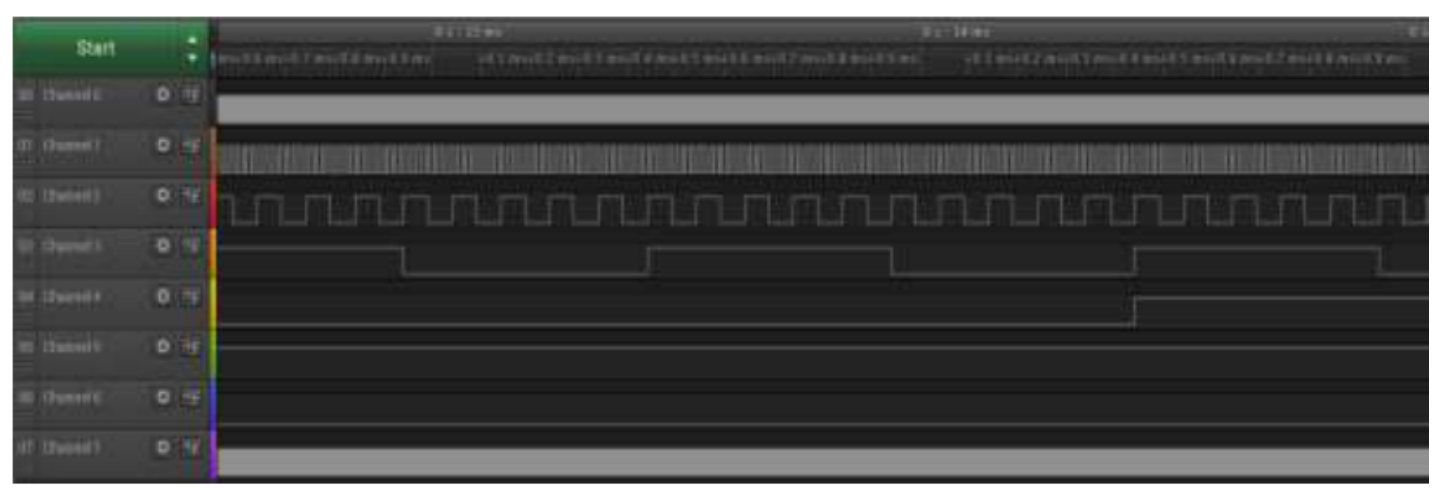

Figure $16100 \mathrm{~Hz}$ frequency on Channel 3.

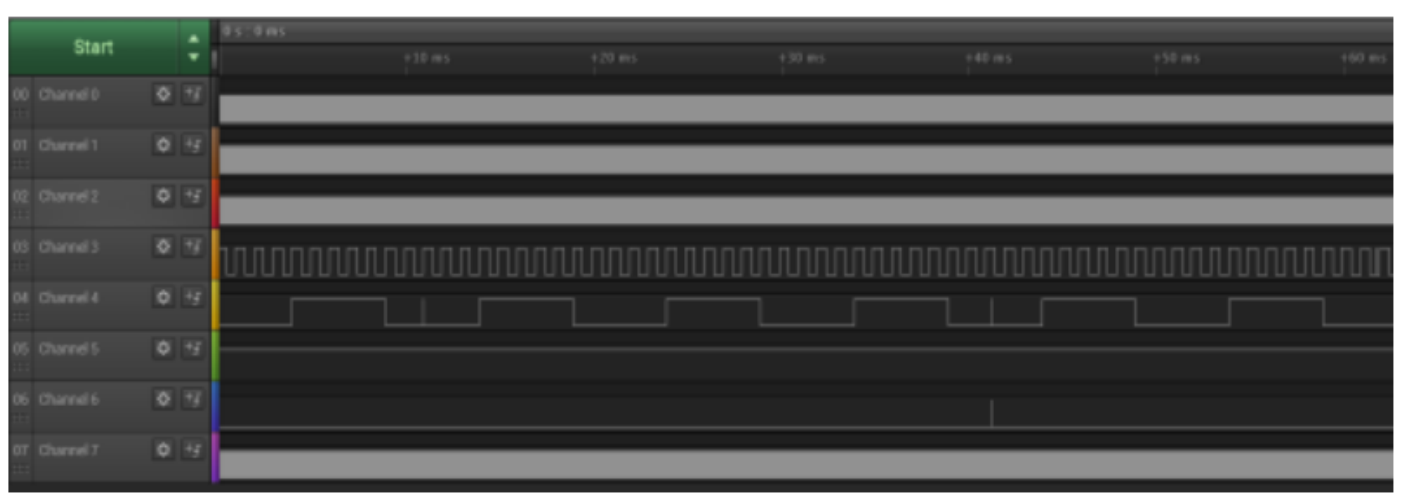

Figure $1710 \mathrm{~Hz}$ frequency on Channel 4.

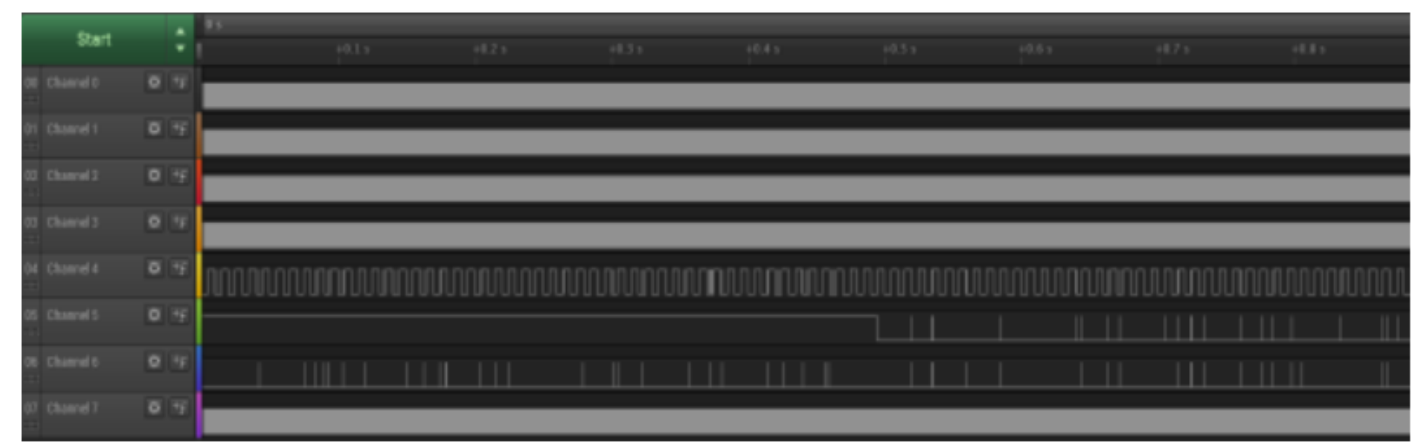

Figure $181 \mathrm{~Hz}$ frequency on Channel 5. 


\section{EXPERIMENTAL SETUP TO TEST DPG AND DLA}

The following figure 19 shows the research experimental setup to test both DPG and DLA using a processor core as CUT. DPG, processor core and DLA all will be implemented in an FPGA.

- Programmable DPG output will load asynchronous controls and synchronous data/address/clock to the processor core, depending on the type of instructions of the processor to be tested.

- The processor core will execute the instructions enabled by the DPG output.

- Data bus \& address bus can be of any width (8/16/32/64/128/256 etc.).

- The processor will output the data (results) to our proposed DLA, whose functionality was explained earlier [12], [13].

- The same processor output data is also applied to DLA product.

- LCD will provide processor status which includes input, output data, Opcode and output results.

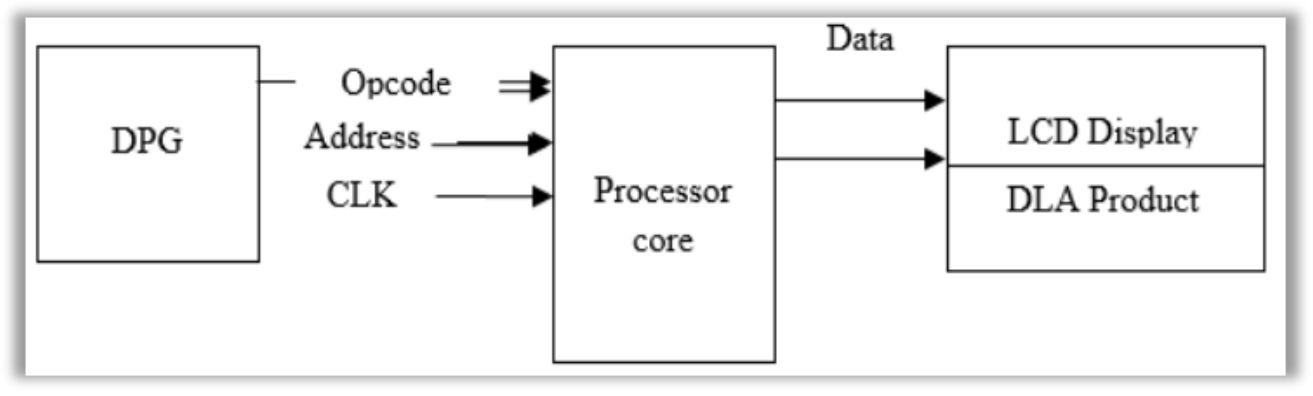

Figure 19 Experimental setup to test both DPG and DLA using a processor core.

\section{FPGA ATE BREAKTHROUGH}

- Programmability for DLA and DPG

- Re-programmability and design up-gradation

- Scalability up to $1000 \mathrm{I} / \mathrm{Os}$

- Speed up to $1 \mathrm{GHz}$ measurement possible

- Feasible synchronization between processor speed and display

- FPGA provides programmable memory width and depth.

- PROGRAMMABLE DPG and DLA CHANNELS- 8/16/32/64...256..1024 channels

- Non real time high speed buffered and programmable DLA

- CUT based customization possible for both DLA and DPG

- FPFA for implementation of fully programmable scalable ATE

\section{CONCLUSION}

In this paper, two innovative designs are explained Processor Clock signal presence Detector using Edge Detector. FCSRE is used to verify the functionality of Processors and Memories, by using the already designed and implemented multi-channel and programmable DPG. The results are tabulated in terms of incoming clock frequency, maximum binary count and number of LEDs glowing along with the simulation waveform of all the sub modules. The FPGA demo snapshots of FCSRE are also shown. A DLA test methodology is also illustrated 
FPGA Based Clock Frequency Sensor and Range Estimating System for Testing Memories and

Processors

along with the DLA waveform screenshots to double verify the frequency ranging and estimation results. The final research experiment setup is described in detail along with the major breakthrough of overall research.

\section{REFERENCES}

[1] T. S Rathore, (2003.) "Digital Measurements Techniques", $2^{\text {nd }}$ edition, Narosa publishing house

[2] George C Barney, (1988) "Intelligent Instrumentation: Microprocessor Applications in measurement and control", Prentice Hall

[3] F. Kraja and G. Acher, (2011) "Using many-core processors to improve the performance of space computing platforms", In 2011 IEEE Aerospace Conference, pp. 1-17

[4] M. Berg, (2009) "Embedding asynchronous FIFO memory blocks in Xilinx Virtex series FPGAs targeted for critical space system applications", in Proceedings of the Military/Aerospace PLD Conference

[5] Tilera, "Tilera Processors," Website, February (2012). Available: http://www.tilera.com/products/processors.

[6] by Gabriel J. García ",Carlos A. Jara, Jorge Pomares, Aiman Alabdo, Lucas M. Poggi and Fernando Torres (2014) A Survey on FPGA-Based Sensor Systems: Towards Intelligent and Reconfigurable", Low-Power Sensors for Computer Vision, Control and Signal Processing, 14(4), pp. 6247-6278

[7] Dondo, J.; Molina, F.S.; Rincon, F.; Moya, F.; Lopez, J.C, (2011) "Ubiquitous FPGA Access for Data Intensive Computing." In Proceedings of the International Symposium on Ubiquitous Computing and Ambient Intelligence, Riviera Maya, Mexico, 5-9, Dec.

[8] Gultekin, G.K.; Saranli, A, (2013) "An FPGA based high performance optical flow hardware design for computer vision applications", Microprocess Microsyst, 37, pp. 270-286

[9] Ahmad Shawahna, Sadiq M. Sait and Aiman El-Malehi, (2018) "FPGA-based Accelerators of Deep Learning Networks for Learning and Classification: A Review", IEEE Access

[10] ALS FPGA trainer kit manual.

[11] https://www.scilab.org/signal-edge-detection. [Accessed Online: 15.07.2020]

[12] https://www.sciencedirect.com/topics/computer-science/frequency-measurement. [Accessed Online: 15.07 .2020$]$

[13] www.xilinx.com. [Accessed Online: 15.07.2020]

[14] P Vijayakumar, T Rajendran, G R Mahendra Babu (2017) Efficient Implementation of Decoder using Modified Soft Decoding Algorithm in Golay $(24,12)$ Code. Pakistan Journal of Biotechnology, 14(Special issue II), pp. $200-203$

[15] S Keerthivasan, G R Mahendra Babu, T Rajendran (2017) Design of Low Intricate 10-Bit Current Steering Digital to Analog Converter Circuitry using Full Swing GDI. Pakistan Journal of Biotechnology, 14(Special issue II), pp. $204-208$

[16] Muthu Felix, T. S. Aravinth, T. Rajendran (2017) Design of CMOS 8-Bit Parallel Adder Energy Efficient Structure using SRCPL Logic Style. Pakistan Journal of Biotechnology, 14(Special issue II), pp. $257-260$

[17] P. Yuvaraj, T. Rajendran, Subramaniam Kamalraj (2017) Design of 4-Bit Multiplexer using Sub-Threshold Adiabatic Logic (STAL). Pakistan Journal of Biotechnology, 14(Special issue II), pp. $261-264$ 
[18] Alahari Radhika, K. Satya Prasad, K. Kishan Rao (2020) High Performance RNS for QR Decomposition Matrix Inversion Architecture. Journal of Critical Reviews, 7 (10), pp. 251255

[19] Sushanta Kumar Mandal, Shailendra Tripathi (2020) Edge Detection using Sobel Technique. Journal of Critical Reviews, 7 (10), pp. 929-933

[20] Sweety, Prachi Chaudhary (2020) Design and FPGA Based Implementation of 1-BIT Dynamic Branch Predictor for the Parallelism Processor. Journal of Critical Reviews, 7 (9), pp. $1156-1162$ 